\title{
OBITUARY: MARCEL F. NEUTS 1935-2014
}

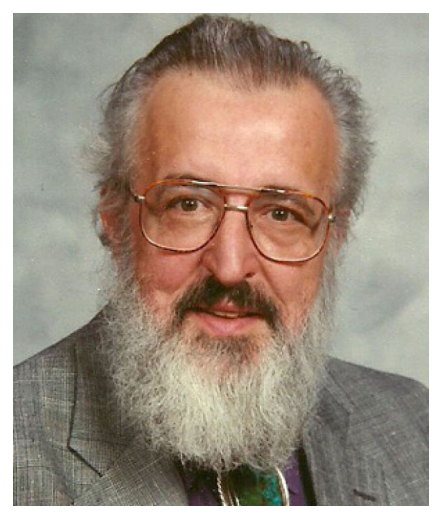

Marcel F. Neuts 1935-2014

Marcel Neuts died in his home in Tucson, Arizona, on 9 March 2014. He was born in Belgium on 21 February 1935, and received his school and undergraduate education in Belgium before moving to Stanford University for his Masters (1958-1959) and PhD (1959-1960), supervised by Samuel Karlin. His main academic appointments were at Purdue University (1962-1976), University of Delaware (1976-1985), and The University of Arizona from 1985 until his retirement in 1996.

Marcel Neuts' early papers from the 1960s largely deal with questions from classical applied probability and incorporate topics such as game theory, extreme value theory, and Markov renewal methods. Two keywords for his later work are structure and computability. His first major contribution in these directions was the formulation of the concept of phase-type (PH) distributions, the pioneering paper being [3]. Here a PH random variable $T$ is defined in terms of a finite continuous-time Markov process $J(t)$ with one absorbing state $\Delta$, with $T=\inf \{t>0: J(t)=\Delta\}$ the time to absorption in $\Delta$. This framework comprises examples such as the mixture or convolutions of $p$ exponentials (of which there is an abundance of examples in earlier literature with $p=2$ ) and many more general ones. Two main advantages are denseness (any distribution on $(0, \infty)$ can be approximated arbitrarily well in the weak sense by a PH distribution with sufficiently large $p$ ); and computational tractability in applications. Initially, the applications were mainly in queueing, but PH distributions have later appeared in many other areas.

The motivation for this work came to a large extent from Marcel Neuts' interest in queues with semi-Markov services or arrivals. With PH assumptions, these can be viewed as countable state Markov chains/processes $(L(t), J(t))$ with transition matrices/generators of a special block structure of one of the following forms:

$$
\left(\begin{array}{ccccc}
\boldsymbol{B}(0) & \boldsymbol{F}(1) & \mathbf{0} & \mathbf{0} & \ldots \\
\boldsymbol{B}(1) & \boldsymbol{F}(0) & \boldsymbol{F}(1) & \mathbf{0} & \ldots \\
\boldsymbol{B}(2) & \boldsymbol{F}(-1) & \boldsymbol{F}(0) & \boldsymbol{F}(1) & \ldots \\
\boldsymbol{B}(3) & \boldsymbol{F}(-2) & \boldsymbol{F}(1) & \boldsymbol{F}(0) & \ldots \\
\vdots & & & & \ddots
\end{array}\right), \quad\left(\begin{array}{cccccc}
\boldsymbol{A}(0) & \boldsymbol{A}(1) & \boldsymbol{A}(2) & \boldsymbol{A}(3) & \ldots \\
\boldsymbol{F}(-1) & \boldsymbol{F}(0) & \boldsymbol{F}(1) & \boldsymbol{F}(2) & \ldots \\
\mathbf{0} & \boldsymbol{F}(-1) & \boldsymbol{F}(0) & \boldsymbol{F}(1) & \ldots \\
\mathbf{0} & \mathbf{0} & \boldsymbol{F}(-1) & \boldsymbol{F}(0) & \ldots \\
\vdots & & & & \ddots
\end{array}\right)
$$


Here the entries are matrices, and rows/columns correspond to levels (for example, queue lengths) of the $L$ component and the states within blocks to states of $J$. Marcel Neuts developed a systematic theory of such structures, starting with the GI/M/1 type highlighted in his 1981 book [6]. The GI/M/1-type model is named because, in the special case when $J$ has just one state, it is the imbedded Markov chain of the standard GI/M/1 queue. There is a huge number of stochastic models with this structure, which were studied either by Marcel Neuts himself or jointly with students and other collaborators. A cornerstone in his theory is the scheme for the stationary distribution which is matrix geometric with block partitioned probabilities $\boldsymbol{\alpha}, \boldsymbol{\alpha} \boldsymbol{R}, \boldsymbol{\alpha} \boldsymbol{R}^{2}, \ldots$ for a certain row vector $\boldsymbol{\alpha}$ and a certain matrix $\boldsymbol{R}$. Moreover, the matrix $\boldsymbol{R}$ can be characterised as the minimal nonnegative solution to the fixed-point problem

$$
\boldsymbol{R}=\boldsymbol{F}(-1)+\boldsymbol{F}(0) \boldsymbol{R}+\boldsymbol{F}(1) \boldsymbol{R}^{2}+\cdots .
$$

This also provides the computational scheme: iteration, contrasting with previous methods, which relied on complex analysis. The $\mathrm{M} / \mathrm{G} / 1$-type model is in many respects the dual to the GI/M/1-type model and the theory is similar, though more difficult. There is an early summary in [4] and a full treatment in the monumental book [8].

Among the main contributions of Marcel Neuts we mention last but not least the Markovian arrival process (MAP) originating from [5] (see also [2]). It is a point process on the line generated by a finite Markov process $J(t)$, such that there are Poisson arrivals at rate $\lambda_{i}$ on intervals where $J(t)=i$ and then some additional arrivals with probability $G_{i j}$ at jumps $i \rightarrow j$ of $J$; a special case (with $G_{i j}=0$ for all $i$ and $j$ ) is the Markov-modulated Poisson process. The MAP is in many respects the point process analogue of the $\mathrm{PH}$ distribution, it enjoys similar flexibility and denseness properties, and it represents most often a much more natural model than the renewal processes dominant in earlier non-Poissonian modeling attempts.

An example bringing all these ingredients together is a $\mathrm{MAP} / \mathrm{PH} / 1$ queue: the level component $L$ is the queue length and $J$ is the pair $\left(J_{1}, J_{2}\right)$ with $J_{1}$ giving the Markov state of the arrival process and $J_{2}$ the phase the server is currently operating in (some modifications apply at the boundary level $\ell=0$ ). The model is in fact a QBD (quasi-birth-death process): the generator matrix is of both $\mathrm{GI} / \mathrm{M} / 1$ and $\mathrm{M} / \mathrm{G} / 1$ type, i.e. $\boldsymbol{F}(\ell)=\mathbf{0}$ for $|\ell|>1$; this special class is another favourite of Marcel Neuts and his followers.

There were occurrences of some of these ideas in earlier literature, but they were scattered and not all well known. Marcel Neuts realised their importance, worked out a systematic theory, made the models and methods widely known, and pioneered the computational aspect. In total he authored four books and more than 150 scientific papers.

Marcel Neuts graduated 21 students, several of which have made important contributions to the field of matrix-analytic methods. He had a profound impact on his students and collaborators, both professionally and personally, as is well evidenced by the foreword to the honorary issue [1]. His influence, however, goes far beyond the community of people in his close circle, and few have become as synonymous with a whole research area as he.

Beyond his scientific contributions, Marcel Neuts' legacy is manifest in the series of conferences in the area of matrix-analytic methods. The first of these took place in Flint, Michigan, August 1995, the most recent-MAM8 - in Kozhikode, India, in January 2014; several important contributions to the field have been published in the proceedings. Another important venue for progress in matrix-analytic methods and other applied probability directions is the journal Stochastic Models which was founded by Marcel Neuts, with the first issue appearing in 1985. In addition, he has served on many editorial boards, in particular for JAP/AAP from 1979 to 2004 (of which he was Coordinating Editor from 1993 to 2004). 
Marcel Neuts is survived by Olga, his wife of almost 55 years, and four children. With him, the field of applied probability has lost one of its major figures over several decades.

\section{Aarhus University}

Søren Asmussen

Technical University of Denmark

[1] Chakravarty, S. R., Dudin, A. N., Khrishnamoorty, A., Resnick, S. I., Bhat, U. N., Lucantoni, D., Wolfson, D., Ramaswami, V. and Meier-Hellstern, K. (2011). Foreword to Special Issue in Honor of Professor Marcel F. Neuts. Stoch. Models 27, 555-568.

[2] Lucantoni, D. M., Meier-Hellstern, K. S. and Neuts, M. F. (1990). A single-server queue with server vacations and a class of nonrenewal arrival processes. Adv. Appl. Prob. 22, 676-705.

[3] Neuts, M. F. (1975). Probability distributions of phase type. In Liber Amicorum Professor Emeritus H. Florin, Department of Mathematics, University of Louvain, Belgium, pp. 173-206.

[4] Neuts, M. F. (1979). Queues solvable without Rouché's theorem. Operat. Res. 27, 767-781.

[5] Neuts, M. F. (1979). A versatile Markovian point process. J. Appl. Prob. 16, 764-779.

[6] Neuts, M. F. (1981). Matrix-Geometric Solutions in Stochastic Models: An Algorithmic Approach. Johns Hopkins University Press, Baltimore, MD.

[7] Neuts, M. F. (1986). An algorithmic probabilist's apology. In The Craft of Probabilistic Modelling: A Collection of Personal Accounts (Appl. Prob. Trust Ser.), ed. J. Gani, Springer, New York, pp. 213-221.

[8] Neuts, M. F. (1989). Structured Markov Chains of the M/G/1 Type and Their Applications. Marcel Dekker, New York.

[9] Neuts, M. F. (1995). Algorithmic Probability: A Collection of Problems. Chapmann \& Hall, London. 